\title{
Heavy Flavor Baryon States at the Tevatron
}

\author{
Sally Seidel ${ }^{\mathrm{a}}$ \\ ${ }^{a}$ Department of Physics and Astronomy, University of New Mexico, Albuquerque, NM 87131, USA \\ For the CDF and DO Collaborations
}

\begin{abstract}
Precision measurements of the masses and widths of the bottom baryon resonances $\Sigma_{\mathrm{b}}$ and $\Sigma_{\mathrm{b}}{ }^{*}$ and charm baryons $\Lambda_{\mathrm{c}}(2595), \Lambda_{\mathrm{c}}(2625), \Sigma_{\mathrm{c}}(2455)$, and $\Sigma_{\mathrm{c}}(2520)$ are reported. A new measurement of $\Lambda_{\mathrm{b}}$ production is described. The studies include the first measurement of the widths and isospin mass splittings of the members of the $\Sigma_{b}$ family. The charm baryons are examined through their strong decays to the $\Lambda_{c}$ ground state, and measurements of their mass differences relative to the ground state, and corresponding decay widths, are reported. The data were collected by the CDF and D0 detectors for $1.96 \mathrm{TeV}$ proton-antiproton collisions during Run II at the Fermilab Tevatron.
\end{abstract}

Keywords: Baryon, bottom quark, charm quark, resonance

PACS: $14.20 . \mathrm{Lq}, 14.20 . \mathrm{Mr}$

\section{INTRODUCTION}

The CDF [1] and D0 [2] Experiments, operating at the Fermilab Tevatron Collider, have since 2002 collected large datasets rich in heavy quark events at collision centerof-mass energy $1.96 \mathrm{TeV}$. The past five years have been an especially fruitful period of discoveries and other breakthrough measurements of heavy baryons. Among these are the measurements of the $\Lambda_{b}$ lifetime in the $\Lambda_{b} \rightarrow J / \psi \Lambda^{0}$ [3] and $\Lambda_{b} \rightarrow \Lambda_{c} \pi^{-}$[4] channels, each the single most precise measurement of the lifetime at its time of publication. During the same era the cross section times branching ratio for the decay $\Lambda_{b} \rightarrow \Lambda_{c} \pi^{-}$[5] and the branching ratios of $\Lambda_{b} \rightarrow \Lambda_{c} \pi^{-} \pi^{+} \pi^{-}$[6] and $\Lambda_{b} \rightarrow \Lambda_{c} \mu \nu$ [7] were also measured for the first time. The $\Xi_{\mathrm{b}}{ }^{-}[8,9]$ and $\Sigma_{\mathrm{b}}{ }^{\left({ }^{*}\right)}$ [10] were reported, providing benchmarks against which Heavy Quark Effective Theory can be compared. The $\Omega_{\mathrm{b}}$ was discovered, and its lifetime and relative production rate were measured [11, 12]. Charmless decays of the $\Lambda_{\mathrm{b}}$ were observed [13,14], providing a new window on CP violation and the $\mathrm{CKM}$ matrix element $\mathrm{V}_{\mathrm{ub}}$.

\section{CHARM BARYON SPECTROSCOPY: THE PROPERTIES OF $\Lambda_{\mathrm{C}}(2595), \Lambda_{\mathrm{C}}(2625), \Sigma_{\mathrm{C}}(2455)$, AND $\Sigma_{\mathrm{C}}(2520)$}

Predicting the mass spectrum and spin-dependent energy splittings in the regime of small momentum transfer relevant to the charm baryon family requires non- 
perturbative techniques. Available predictions are based on QCD sum rules, lattice calculations, quark models, and a bag model. This is an area in which experimental data can offer substantial guidance to theoretical development. All of the $\Lambda_{\mathrm{c}}{ }^{*}$ and $\Sigma_{\mathrm{c}}$ states described here are accessible through their strong decays to $\Lambda_{\mathrm{c}}$ plus one or two pions.

The dataset includes $5.2 \mathrm{fb}^{-1}$ acquired by CDF between February 2002 and June 2009. Event reconstruction begins with requirements on the quality of candidate tracks including, for example, the number of hits in the tracker. Tracks that pass are fitted under $\mathrm{K}, \pi$, and $\mathrm{p}$ hypotheses, and $\mathrm{pK} \pi$ vertices are formed for potential $\Lambda_{\mathrm{c}}$ candidates. The proposed proton and pion contributors to the vertex are required to have the same charge, and the total charge of the $\mathrm{pK} \pi$ candidate is required to be \pm 1 . At this point the dataset is divided in half, with one portion being used to train a neural network and the other used in the analysis. Then the roles are reversed, so every event is used both for training and for analysis, with the samples always independent. The network is trained on $\Lambda_{c}$ 's by using events with a displaced secondary vertex, high track transverse momentum $\mathrm{p}_{\mathrm{T}}$, and correct particle identities taken from the time-offlight system and $\mathrm{dE} / \mathrm{dx}$ information. The network is trained on data exclusively.

The distribution of reconstructed masses of $\Lambda_{c}$ candidates in the training sample is fitted to a Gaussian plus linear background. This produces a probability density function that reflects the likelihood that each training event is signal or background on the basis of its proximity to the mean of the Gaussian. This is the sPlot technique[15]. The trained neural network with weights given by the probability distribution functions is applied to the analysis sample, and $\Lambda_{\mathrm{c}}$ candidates are selected on the basis of track opening angles, impact parameter significance, track $\mathrm{p}_{\mathrm{T}}$, track identity likelihood, quality of the vertex reconstruction, and secondary vertex displacement. The next stage is the reconstruction of the $\Sigma_{\mathrm{c}}$ or $\Lambda_{\mathrm{c}}{ }^{*}$. In the training samples for these, the event weights are again based upon the mass difference relative to the Gaussian mean. The network inputs include the charmed baryon proper decay time and vertex quality of fit $\left(\chi^{2}\right)$, the $\Lambda_{c}$ likelihood (taken from the prior network) of being signal, (for the $\Sigma_{\mathrm{c}}$ ) the impact parameter uncertainty and the transverse impact parameter of the daughter pion, and (for the $\Lambda_{c}{ }^{*}$ ) the uncertainty on the impact parameter of the $\pi \pi$ pair. Independent networks are used for charged and neutral $\Sigma_{\mathrm{c}}$ 's. Training is based on the $\Sigma_{\mathrm{c}}(2455)$ and $\Lambda_{\mathrm{c}}{ }^{*}(2625)$, but the networks are applied to the partner states as well. As with the $\Lambda_{c}$, this network is trained on data exclusively. The network output threshold is selected to maximize signal divided by the square root of signal plus background. To eliminate the systematic uncertainty associated with the $\Lambda_{\mathrm{c}}$ mass, the $\Lambda_{\mathrm{c}}{ }^{*}$ and $\Sigma_{\mathrm{c}}$ are extracted through mass difference spectra $\mathrm{m}\left(\Lambda_{\mathrm{c}}{ }^{+} \pi^{-}\right)-\mathrm{m}\left(\Lambda_{\mathrm{c}}{ }^{+}\right) ; \mathrm{m}\left(\Lambda_{\mathrm{c}}{ }^{+} \pi^{+}\right)-\mathrm{m}\left(\Lambda_{\mathrm{c}}{ }^{+}\right) ;$and $\mathrm{m}\left(\Lambda_{\mathrm{c}}^{+} \pi^{+} \pi^{-}\right)-\mathrm{m}\left(\Lambda_{\mathrm{c}}^{+}\right)$. The function used in the fit is a non-relativistic Breit-Wigner for the signal plus a triple Gaussian for the detector resolution.

The $\Lambda_{\mathrm{c}}{ }^{*}(2595)$ requires special treatment. The kinematic threshold for this state is non-negligible because it decays dominantly through $\Sigma_{\mathrm{c}}(2455) \pi$ channels. Consequently a mass-dependent Breit-Wigner function is applied to this fit. The $\Lambda_{\mathrm{c}}{ }^{*}(2595)$ fit includes contributions from the three final states $\Sigma_{\mathrm{c}}(2455)^{0} \pi^{+}$, 
$\Sigma_{\mathrm{c}}(2455)^{++} \pi^{-}$, and $\Sigma_{\mathrm{c}}(2455)^{+} \pi^{0}$. The process of extracting the signal requires fitting simultaneously to all the possible intermediate $\Sigma_{\mathrm{c}}$ channels. From this, the pion coupling $\mathrm{h}_{2}$ can be extracted, as it enters each amplitude through the $\Sigma_{\mathrm{c}} \pi$ vertex.

Backgrounds to this signal include combinatorial assignments lacking a real $\Lambda_{c}$, events combining a real $\Lambda_{c}$ or $\Sigma_{c}$ with random tracks, and feed-down from other $\Lambda_{c}{ }^{*}$ resonances into the $\Sigma_{\mathrm{c}}$ spectrum. The systematic uncertainties derive from detector resolution, the precision of the mass scale through the precision of the magnetic field, the model used in the fit, and the uncertainty on the world average $\Sigma_{\mathrm{c}}$ mass. The resolution is estimated with Monte Carlo events and validated by data from the kinematically analogous channels $\mathrm{D}^{*}(2010)^{+} \rightarrow \mathrm{D}^{0} \pi^{+}$and $\psi(2 \mathrm{~S}) \rightarrow \mathrm{J} / \psi \pi^{+} \pi^{-}$. The results of the study [16] are summarized in Table 1.

TABLE 1. Masses and widths measured for the $\Sigma_{\mathrm{c}}$ and $\Lambda_{\mathrm{c}}{ }^{*}$ resonances.

\begin{tabular}{lcc}
\hline Resonance & Mass $\left(\mathbf{M e V} / \mathbf{c}^{2}\right)$ & Width $\left(\mathbf{M e V} / \mathbf{c}^{2}\right)$ \\
\hline$\Lambda_{\mathrm{c}}(2595)^{+}$ & $2592.25 \pm 0.24 \pm 0.14$ & $2.59 \pm 0.30 \pm 0.47$ \\
$\Lambda_{\mathrm{c}}(2625)^{+}$ & $2628.11 \pm 0.13 \pm 0.14$ & $<0.97 @ 90 \% \mathrm{CL}$ \\
$\Sigma_{\mathrm{c}}(2455)^{0}$ & $2453.74 \pm 0.12 \pm 0.14$ & $1.65 \pm 0.11 \pm 0.49$ \\
$\Sigma_{\mathrm{c}}(2520)^{0}$ & $2519.34 \pm 0.58 \pm 0.14$ & $12.51 \pm 1.82 \pm 1.37$ \\
$\Sigma_{\mathrm{c}}(2455)^{++}$ & $2453.90 \pm 0.13 \pm 0.14$ & $2.34 \pm 0.13 \pm 0.45$ \\
$\Sigma_{\mathrm{c}}(2520)^{++}$ & $2517.19 \pm 0.46 \pm 0.14$ & $15.03 \pm 2.12 \pm 1.36$ \\
\hline
\end{tabular}

The pion constant $\mathrm{h}_{2}{ }^{2}$ is found to be $0.36 \pm 0.04 \pm 0.07$. This is used to compute the $\Lambda_{\mathrm{c}}(2595)^{+}$width for comparisons with other measurements. The $\Lambda_{\mathrm{c}}(2595)^{+}$mass is 3.1 $\mathrm{MeV} / \mathrm{c}^{2}$ lower than previous measurements because of the inclusion of the threshold effects. The $\Lambda_{\mathrm{c}}(2625)$ result is consistent with previous measurements but significantly more precise. When compared to two previous measurements by CLEO, this mass for the $\Sigma_{\mathrm{c}}(2520)^{++}$favors the earlier one. The results for the $\Sigma_{\mathrm{c}}(2455)^{+}$, $\Sigma_{\mathrm{c}}(2520)^{0}$, and $\Sigma_{\mathrm{c}}(2455)^{0}$ are all consistent with previous measurements.

\section{PRECISION MEASUREMENT OF THE WIDTHS AND MASSES OF BOTTOM BARYON RESONANCES $\Sigma_{\mathbf{b}}$ AND $\Sigma_{\mathbf{b}}{ }^{*}$}

With heavy quark content $b q_{1} q_{2}$, the $\Sigma_{\mathrm{b}}$ and $\Sigma_{\mathrm{b}}{ }^{*}$ probe non-perturbative QCD in a unique regime. Mass spectra for this family are predicted by Heavy Quark Effective Theory, potential models, and lattice calculations. The isospin mass splittings are expected on theoretical grounds to be dominated by the $u$ - $d$ mass difference. As prediction of the resonance widths is challenging, these data can contribute significantly to the evolution of the theory.

The states are reconstructed through their decay to $\Lambda_{\mathrm{b}}$. A $6.0 \mathrm{fb}^{-1}$ dataset collected from March 2002 to February 2010 is used. The analysis [17] begins with application of quality cuts to all tracks. Next, with no requirement on particle identification, groups of three tracks are fit to a common vertex which is then constrained to the $\Lambda_{c}$ 
mass. A fourth track is added as a pion to this vertex. To minimize combinatorial background, requirements are placed on the tracks' transverse momenta $\mathrm{p}_{\mathrm{T}}$. The $\Lambda_{\mathrm{b}}$ impact parameter $\mathrm{d}_{0}$ magnitude is required to be less than $80 \mu \mathrm{m}$. For consistency, two or more of the tracks must be found by the Silicon Vertex Trigger, which identifies their origin as a displaced vertex. Through a requirement on $c \tau$, the $\Lambda_{b}$ decay vertex is required to be displaced from the primary vertex. The $\Lambda_{\mathrm{b}}$ momentum vector must point back to the primary vertex. At this point a fifth track, assumed to be a pion, is added to the candidate $\Lambda_{\mathrm{b}}$ vertex.

All requirements on the $\Lambda_{b}$ reconstruction are taken from data, optimizing signal divided by the square root of signal plus background. A total of 16300 candidates are obtained. Backgrounds to this reconstruction include combinatorics, partially reconstructed B mesons producing $\Lambda_{\mathrm{c}} \pi$, fully reconstructed B meson decays whose daughter tracks are mis-reconstructed as $\Lambda_{\mathrm{c}} \pi$, partially reconstructed $\Lambda_{\mathrm{b}}$ decays, and fully reconstructed $\Lambda_{b}$ decays to other channels, for example $\Lambda_{b} \rightarrow \Lambda^{0} \mathrm{~K}^{-}$. To minimize the systematic uncertainty associated with the $\Lambda_{\mathrm{b}}$ mass resolution when reconstructing the $\Sigma_{b}$, a fit is made to the mass difference $m\left(\Lambda_{b} \pi\right)-m\left(\Lambda_{c} \pi\right)-m_{\pi}{ }^{\text {PDG }}$. The fit of the signal uses a non-relativistic Breit-Wigner function, with width modified to reflect the pion's p-wave production, convoluted with a double Gaussian resolution function. The background is fitted with a second-order polynomial multiplied by a square root kinematic threshold factor. Separate negative log likelihood functions are constructed for the states $\Sigma_{\mathrm{b}}{ }^{+}$and $\Sigma_{\mathrm{b}}{ }^{{ }^{+}}$and for the states $\Sigma_{\mathrm{b}}{ }^{-}$and $\Sigma_{\mathrm{b}}{ }^{{ }_{-}}$. Each of the four signal peaks is found with significance exceeding 7.0 standard deviations. The systematic uncertainties include the tracker momentum scale (which dominates the mass measurements), the two-Gaussian resolution model (which dominates the width measurements), the model of the background, and the algorithm used to fit the Monte Carlo events. The magnitudes of the uncertainties are validated by comparison with analogous charm decays in the data. The mass difference measurements are combined with the CDF measurement of the $\Lambda_{\mathrm{b}}$ mass, $5609.7 \pm 1.2 \pm 1.2 \mathrm{MeV} / \mathrm{c}^{2}$, to obtain the mass of each of the resonances. These, along with the first measurements of the widths, are shown in Table 2.

TABLE 2. Masses and widths measured for the $\Sigma_{\mathrm{b}}$ and $\Sigma_{\mathrm{b}}{ }^{*}$ resonances.

\begin{tabular}{llc}
\hline Resonance & Mass $\left(\mathbf{M e V} / \mathbf{c}^{2}\right)$ & Width $\left(\mathbf{M e V} / \mathbf{c}^{2}\right)$ \\
\hline$\Sigma_{\mathrm{b}}{ }^{+}$ & $5811.2_{-0.8}^{+0.9} \pm 1.7$ & $9.2_{-2.9-1.1}^{+3.8+1.0}$ \\
$\Sigma_{\mathrm{b}}{ }^{-}$ & $5815.5_{-0.5}^{+0.6} \pm 1.7$ & $4.3_{-2.1 .1 .1}^{+3.1+1.0}$ \\
$\Sigma_{\mathrm{b}}{ }^{+}$ & $5832.0 \pm 0.7 \pm 1.8$ & $10.4_{-2.2-1.2}^{+2.7+0.8}$ \\
$\Sigma_{\mathrm{b}}{ }^{-}$ & $5835.0 \pm 0.6 \pm 1.8$ & $6.4_{-1.8-1.1}^{+2.2+0.7}$ \\
\hline
\end{tabular}

The mass difference measurements improve upon the precision of previous measurements by better than a factor of two. The isospin mass splittings are now available for the first time, at precision comparable to those of charm states. The negative isospin states are found to have mass slightly higher than the positive ones, contrary to the case for charm. The natural widths are in agreement with theoretical predictions. 


\section{MEASUREMENT OF THE PRODUCTION FRACTION TIMES BRANCHING FRACTION $f\left(b \rightarrow \Lambda_{b}\right) \cdot B\left(\Lambda_{b} \rightarrow J / \psi \Lambda\right)$}

Decays of $\mathrm{b}$ hadrons may be a window onto physics beyond the Standard Model. Few measurements of $b$ baryons are available, and uncertainties on their branching fractions are typically $30 \%$ to $60 \%$ or more. Improved experimental precision can be input to pQCD and quark models. A dataset of size $6.1 \mathrm{fb}^{-1}$ has been used by D0 to reconstruct two kinematically similar channels, $\Lambda_{b} \rightarrow J / \psi(\rightarrow \mu \mu) \Lambda\left(\rightarrow p \pi^{-}\right)$and $\mathrm{B}^{0} \rightarrow \mathrm{J} / \psi\left(\rightarrow \mu^{+} \mu^{-}\right) \mathrm{K}_{\mathrm{s}}\left(\rightarrow \pi^{+} \pi^{-}\right)$. In the ratio of their production fractions times branching fractions, systematics on quark production, luminosity, trigger efficiencies, and selection efficiencies cancel.

The method for selecting these events begins with application of quality cuts to tracks, including the requirements that at least one proton-antiproton collision occurred, a minimum number of hits was present in the tracking system, and the number of hits between the primary and secondary vertices is below an upper bound. The trigger requires 1 or 2 muons. Muons are reconstructed with segments that match from central tracking to the muon detectors. A selection is made on $p_{T}$ and central $\eta$. At least one muon must be observed both inside and outside the toroid. At the next step, pairs of muons are vertexed, and $\mathrm{J} / \psi$ candidates in the mass range $2.8<\mathrm{M}_{\mu \mu}<$ $3.35 \mathrm{GeV} / \mathrm{c}^{2}$ are retained. In events that include these $\mathrm{J} / \psi$ candidates, all other pairs of oppositely charged tracks are vertexed and examined for combinations that may be identified as $\Lambda\left(\mathrm{K}_{\mathrm{s}}\right)$ candidates within the mass range $1.102(0.466)<\mathrm{M}<1.130(0.530)$ $\mathrm{GeV} / \mathrm{c}^{2}$. The impact parameters of these candidates must exceed a minimum value. Feed-down is minimized through a cut on the angle between the $\Lambda \mathrm{p}_{\mathrm{T}}$ and the $\Lambda$ momentum vector. The dimuon is then constrained to the $\mathrm{J} / \psi$ mass, $3.097 \mathrm{GeV} / \mathrm{c}^{2}$. The $\Lambda\left(\mathrm{K}_{\mathrm{s}}\right)$ is vertexed with the dimuon parent to form the $\Lambda_{\mathrm{b}}\left(\mathrm{B}^{0}\right)$ candidate, and that candidate's $\mathrm{p}_{\mathrm{T}}$ is required to exceed a minimum. Finally, the mass of the $\Lambda_{b}\left(\mathrm{~B}^{0}\right)$ is required to lie in the range $5.0(4.8)<\mathrm{M}<6.2(5.8) \mathrm{GeV} / \mathrm{c}^{2}$.

The number of background events is predicted from the sidebands in the data. The number of signal events $\left(\Lambda_{b}\right.$ or $\left.\mathrm{B}^{0}\right)$ is predicted from Monte Carlo simulation. The ratio of signal to the square root of signal plus background is maximized by applying requirements on $\mathrm{p}_{\mathrm{T}}$, transverse decay length and its significance, proper decay length significance, and vertex quality. If a $\Lambda$ and a $\mathrm{K}_{\mathrm{s}}$ are found in the same event, the one with the best vertex is used if they are formed from different tracks, and the event is removed if they are formed from the same tracks. The number of signal events is extracted from an unbinned fit to a double Gaussian plus second order polynomial. The observed yields, $\mathrm{N}\left(\Lambda_{\mathrm{b}} \rightarrow \mathrm{J} / \psi \Lambda\right)=314 \pm 29$ and $\mathrm{N}\left(\mathrm{B}^{0} \rightarrow \mathrm{J} / \psi \mathrm{K}_{\mathrm{s}}{ }^{0}\right)=2335 \pm 73$, are input

$\sigma_{r e l} \equiv \frac{f\left(b \rightarrow \Lambda_{b}\right) \cdot B\left(\Lambda_{b} \rightarrow J / \psi \Lambda\right)}{f\left(b \rightarrow B^{0}\right) \cdot B\left(B^{0} \rightarrow J / \psi K_{s}^{0}\right)}=\frac{N\left(\Lambda_{b} \rightarrow J / \psi \Lambda\right)}{N\left(B^{0} \rightarrow J / \psi K_{s}^{0}\right)} \cdot \frac{B\left(K_{s}^{0} \rightarrow \pi^{+} \pi^{-}\right)}{B\left(\Lambda \rightarrow p \pi^{-}\right)} \cdot \varepsilon$, where 
$\varepsilon \equiv \frac{\varepsilon\left(B^{0} \rightarrow J / \psi K_{s}^{0}\right)}{\varepsilon\left(\Lambda_{b} \rightarrow J / \psi \Lambda\right)}=2.37 \pm 0.05$ is the ratio of efficiencies, with uncertainty deriving from Monte Carlo statistics, $B\left(K_{s}^{0} \rightarrow \pi^{+} \pi^{-}\right)=0.6920 \pm 0.0005$, and $B\left(\Lambda \rightarrow p \pi^{-}\right)=0.639 \pm 0.005$. Uncertainties on the measurement derive from the $\Lambda_{\mathrm{b}}$ and $\mathrm{B}^{0}$ yields $(5.5 \%)$, input from the simulation model to $\varepsilon(2 \%)$, contamination of the $\Lambda_{\mathrm{b}}$ signal by $\mathrm{B}^{0}$ and vice versa $(2.3 \%)$, and $\Lambda_{\mathrm{b}}$ polarization effects upon $\Lambda$ emission (7.2\%). The combined uncertainty is $9.6 \%$. The final result [18] is $\sigma_{\text {rel }}=0.345 \pm$ 0.034 (stat.) \pm 0.033 (syst.) \pm 0.003 (PDG). No variation is found to be correlated with temporal selection, $\eta, p_{\mathrm{T}}$, decay lengths, or other parameters of the events. Monte Carlo events were compared to and confirmed by data for decay length measurements, vertex $\chi^{2}$ distributions, and $\Lambda$ and $\mathrm{K}_{\mathrm{s}}$ lifetime measurements.

\section{SUMMARY AND CONCLUSIONS}

The $\Lambda_{\mathrm{c}}(2595), \Lambda_{\mathrm{c}}(2625), \Sigma_{\mathrm{c}}(2455)$, and $\Sigma_{\mathrm{c}}(2520)$ masses and widths have been measured by CDF to generally improved precision and, for $\Lambda_{c}(2595)$, a significant revision of the world average mass. The pion coupling $h_{2}$ was obtained at the same time. All four $\Sigma_{\mathrm{b}}{ }^{(*)}$ states have been reconfirmed by CDF at significance $>7 \sigma$, and the precision on their masses is improved by more than a factor of 2 . Widths and isospin mass splittings for the four $\Sigma_{\mathrm{b}}{ }^{(*)}$ states have been measured for the first time. The $\Lambda_{\mathrm{b}}$ production cross section relative to $\mathrm{B}^{0}$, times branching fractions to kinematically similar final states, has been measured by D0.

\section{REFERENCES}

1. D.E. Acosta et al. (CDF Collaboration), Phys. Rev. D71, 032001 (2005).

2. V.M. Abazov et al. (D0 Collaboration), Nucl .Instr. and Meth. A 565 (2006) 463.

3. A. Abulencia et al. (CDF Collaboration), Phys. Rev. Lett. 98:122001 (2007).

4. T. Aaltonen et al. (CDF Collaboration), Phys. Rev. Lett. 104:102002 (2010).

5. A. Abulencia et al. (CDF Collaboration), Phys. Rev. Lett. 98:122002 (2007).

6. The CDF Collaboration, "First observation and measurement of the relative branching fractions of charm resonant decay modes in the $\Lambda^{0} \rightarrow \Lambda_{\mathrm{c}}^{+} \pi^{-} \pi^{+} \pi^{-}$decay," Public Note 10001, 6 November 2009.

7. T. Aaltonen et al. (CDF Collaboration), Phys. Rev. D79:032001 (2009).

8. V.M. Abazov et al. (D0 Collaboration), Phys. Rev. Lett. 99:052001 (2007).

9. T. Aaltonen et al. (CDF Collaboration), Phys. Rev. Lett. 99:052002 (2007).

10. T. Aaltonen et al. (CDF Collaboration), Phys. Rev. Lett. 99:202001 (2007).

11. V.M. Abazov et al. (D0 Collaboration), Phys. Rev. Lett. 101:232002 (2008).

12. T. Aaltonen et al. (CDF Collaboration), Phys. Rev. D80:072003 (2009).

13. T. Aaltonen et al. (CDF Collaboration), Phys. Rev. Lett. 106:181802 (2011).

14. T. Aaltonen et al. (CDF Collaboration), Phys. Rev. Lett. 103:031801 (2009).

15. M. Pivk and F.R. Le Diberder, Nucl. Instr. and Meth. A555, 356 (2005).

16. T. Aaltonen et al. (CDF Collaboration), arXiv:1105.5995 [hep-ex].

17. C. Calancha and I. Gorelov, PoS ICHEP2010 (2010) 203 and arXiv:1105.5995 [hep-ex].

18. V.M. Abazov, et al. (D0 Collaboration), arXiv:1105.0690 [hep-ex]. 